\title{
Expression of Wnt3a in hepatocellular carcinoma and its effects on cell cycle and metastasis
}

\author{
CAIJIE LU ${ }^{1 *}$, YIFENG HE $^{1 *}$, JUAN DUAN $^{2 *}$, YONGGUANG YANG ${ }^{1}$, CHUNQIANG ZHONG $^{1}$, \\ JIAN ZHANG ${ }^{1}$, WEIGUO LIAO ${ }^{1}$, XIAOJIE HUANG ${ }^{1}$, RUNZHI ZHU ${ }^{1}$ and MINGYI LI ${ }^{1}$ \\ ${ }^{1}$ Laboratory of Hepatobiliary Surgery, Affiliated Hospital of Guangdong Medical University, \\ Zhanjiang Key Laboratory of Hepatobiliary Diseases; ${ }^{2}$ Guangdong Medical University, \\ Zhanjiang, Guangdong 524001, P.R. China
}

Received June 20, 2017; Accepted August 28, 2017

DOI: 10.3892/ijo.2017.4112

\begin{abstract}
Invasion and metastasis are the primary causes of mortality from hepatocellular carcinoma (HCC). Effective inhibition against participants in the tumourigenesis and metastasis process is critical for treatment of HCC. Wnt3a is involved in the development and metastasis of many malignant tumours. However, the specific mechanisms of Wnt3a-mediated cell proliferation, invasion and metastasis in $\mathrm{HCC}$ remain unclear. In this study, we found that Wnt $3 \mathrm{a}$ and its target gene c-Myc showed higher expression in tumour tissues than normal liver tissues in HCC patients; $71.8 \%$ of the cases studied had high Wnt3a and c-Myc expression levels $(n=32)$; Wnt3a expression positively correlated with its target genes MMP-7 and c-Myc. Intriguingly, the expression of Wnt3a, MMP-7 and c-Myc is significantly correlated with Notch3 and Hes1 expression. In vitro experiments showed that Wnt3a was highly expressed in $\mathrm{MHcc} 97 \mathrm{H}$ and SK-Hep-1 cells. Therefore, Wnt3a expression was silenced with siRNA, and then, MTT, flow cytometry, wound healing and Transwell assays were performed to analyse cell proliferation, cycle, migration and invasion. The results demonstrated that downregulation of Wnt3a expression inhibited cell viability and induced $G_{0} / G_{1}$ cell cycle arrest via decreased expression of cyclin D1 and $\mathrm{c}-\mathrm{Myc}$ and increased expression of p21 and p27. In addition, deletion of Wnt3a significantly inhibited migration and invasion by downregulating MMP-2/-7/-9 expression via the MAPK (p38, ERK1/2 and JNK) pathway. In conclusion, our
\end{abstract}

Correspondence to: Dr Runzhi Zhu or Dr Mingyi Li, Laboratory of Hepatobiliary Surgery, Affiliated Hospital of Guangdong Medical University, Zhanjiang Key Laboratory of Hepatobiliary Diseases, 57 South Renmin Road, Zhanjiang, Guangdong 524001, P.R. China E-mail: hepatolab@126.com

E-mail: limingyi62@163.com

*Contributed equally

Key words: Wnt3a, migration, invasion, MMPs, MAPK pathway, hepatocellular carcinoma data show that Wnt3a is involved in HCC development. Wnt3a may be an effective target for treatment of HCC.

\section{Introduction}

Hepatocellular carcinoma (HCC) is the third most common cancer worldwide, and over $50 \%$ of patients emerge from Asia (1). Although significant progress has been made in the diagnosis and treatment of $\mathrm{HCC}$, the overall prognosis is still poor, and high metastasis and recurrence rates are the main factors affecting the prognosis of HCC patients $(2,3)$. Therefore, it is important to investigate and clarify the molecular mechanisms underlying HCC metastasis and recurrence in order to find novel therapeutic targets.

The Wnt signalling pathway is a highly conserved signalling pathway that controls cell growth, differentiation, apoptosis and self-renewal, and it is implicated in maintenance of tumour progenitor cells, drug resistance, tumour progression, invasion and metastasis (4,5). Canonical Wnt signalling is mediated by ligand binding to Frizzled and the low-density lipoprotein receptor-related protein-5/6 (LRP5/6) receptors, resulting in the accumulation of $\beta$-catenin in the cytoplasm and $\beta$-catenin transfer to the nucleus, where it interacts with $\mathrm{T}$ cell factor (TCF)/lymphatic enhancement factor (LEF) to regulate the expression of various target genes $(6,7)$. Wnt $3 \mathrm{a}$ is a ligand in the Wnt signalling pathway. It has been reported that Wnt3a is activated and participates in the metastasis process in many cancers, including non-small cell lung cancer, colorectal cancer, gastric cancer and breast cancer (8-11). Recently, some researchers have suggested that targeting the $\mathrm{Wnt} / \beta$-catenin signalling pathway can regulate $\mathrm{HCC}$ cell proliferation, invasion and metastasis (12-14). However, these studies primarily focused on $\beta$-catenin, and whether Wnt3a is activated and involved in the occurrence and metastasis of HCC has rarely been reported.

Given the effects of Wnt3a on progression, recurrence and metastasis of other tumours, it is important to analyse the function of Wnt3a in HCC. In the present study, we assessed the critical role of Wnt3a in HCC. We also investigated the effects of Wnt3a siRNA on cell cycle distribution and the metastatic ability of HCC cells in vitro. Our findings suggest novel therapeutic strategies for HCC treatment. 


\section{Materials and methods}

Clinical specimens. In total, 32 primary HCC patients, diagnosed by an oncologist and a pathologist, were sampled from a prospectively designed database. In accordance with the protocol approved by the Affiliated Hospital of Guangdong Medical University (Ethics Committee protocol no. PJ2013105), all the participants signed an informed consent. We ensured that the patients understood the information regarding the application of specimens. Cancerous tissues and adjacent tissues from 32 patients were obtained from the Hepatobiliary Surgery Center and were frozen in liquid nitrogen before use. None of the patients received radiotherapy or chemotherapy before sample collection.

Cell culture. Several hepatoma cell lines (SK-HEP-1, QGY7701, HeP3B, QGY7703, HepG2, MHCC97H, MHCC97L, and Smmc7721) and one immortalized liver cell line (HL7702) were used in this study. All cells were purchased from the Cell Bank of Shanghai (China). The HepG2, HL7702 and Smmc7721 cells were cultured in RPMI-1640 basic medium supplemented with 10\% fetal bovine serum (FBS, cat. no. 10100-147; Gibco, Australia). The other cells were cultured in DMEM supplemented with $10 \%$ FBS.

siRNA transfection. MHcc97H and SK-Hep-1 cells were plated into 6-well plates and transfection procedures were performed according to the manufacturer's protocol. To confirm the validity of the experiment, we screened the efficacy of Wnt3a knockdown and selected the best performing siRNA transcript. Before each experiment, we transiently transfected MHcc97H and SK-Hep-1 cells using an siRNA-Wnt3a gene knockdown kit (Jima Biotechnology Co., Shanghai, China) and the negative control siRNA (NC-si).

Cell inhibition and cytotoxicity assay (MTT assay). $\mathrm{MHcc} 97 \mathrm{H}$ and SK-Hep-1 cells ( $1 \times 10^{4}$ cells/well) were seeded into 96-well plates. Following $24 \mathrm{~h}$ of adherent culture at $37^{\circ} \mathrm{C}$, the cells were treated with siRNA to inhibit Wnt3a expression. Phosphatebuffered saline treatment served as a blank control, and NC-si treatment acted as a negative control. Then, $20 \mu \mathrm{l}$ of MTT stock solution at a concentration of $5 \mathrm{mg} / \mathrm{ml}$ was transferred to each well to obtain a final volume. Next, the cell supernatants were gently removed, and $200 \mu \mathrm{l}$ of DMSO was added to each well to solubilize the formed crystals. The absorbance was detected at $570 \mathrm{~nm}$ with a spectrophotometer (Perkin-Elmer, USA).

Flow cytometry analysis. The cells treated with siRNA were

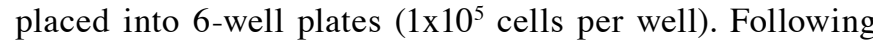
overnight growth, cells were carefully collected, pelleted, washed with PBS, and suspended in binding buffer. Then, $100 \mathrm{ml}$ of cells was incubated with $5 \mathrm{ml}$ of Annexin V-FITC and $5 \mathrm{ml}$ of PI for $15 \mathrm{~min}$ in the dark. The fixed cells under each experimental condition were examined, and DNA content was analysed using FlowJo software. Then, cell cycle analysis was performed with a flow cytometer (BD FACSCalibur). All measurements were performed as described under the same instrumental settings following the manufacturer's protocol.

Colony formation assay. Briefly, MHcc97H and SK-Hep-1 cells were initially plated in 6-well culture dishes (BD) and cultured in DMEM containing 10\% FBS for seven days, and the medium was refreshed every two days. After the incubation, we removed the medium and washed the cells twice with PBS. Then, the cells were stained with $1 \%$ crystal violet for 15 min before being counted. The results were photographed with a camera and analysed with ImageJ software. All the studies were repeated three times.

Wound healing assay. The cells were cultured in a 24-well plate in DMEM containing 10\% FBS for $24 \mathrm{~h}$ until they grew to $100 \%$ confluence. A straight scratch was created in the adherent cells with a pipette tip after three washes with phosphate-buffered saline to remove cellular debris. Then, the cells were cultured in DMEM containing 1\% FBS. The wounds were observed under a microscope, and images were captured at 0,12 and $24 \mathrm{~h}$. The data were analysed with ImageJ software, and the experiments were conducted in triplicate independently.

Transwell assay. MHcc $97 \mathrm{H}$ and SK-Hep-1 cells were seeded in Transwell chambers at a concentration of $1 \times 10^{5}$ cells/chamber in the absence or presence of Wnt3a-siRNA treatment. Transwell inserts (chambers) were placed in a 24 -well plate, cells were diluted with serum-free DMEM, and growth medium (DMEM with 10\% FBS) was added to the lower chambers. After incubation for $24 \mathrm{~h}$ at $37^{\circ} \mathrm{C}$, the cells that passed through the membrane to the bottom wells were fixed in $75 \%$ ethanol and stained with $0.1 \%$ crystal violet (Amresco, Solon, OH, USA). The cells were observed under a microscope and imaged, and the experiments were performed three times independently. Cell invasion ability was also assessed. The cell invasion assay was similar to the cell motility assay, with the exception that the inserts were pretreated with Matrigel (1:10 diluted in DMEM).

$q R T$-PCR analysis. Total RNA was extracted from frozen tissues using TRIzol reagent (Invitrogen, Guangzhou, China) according to the manufacturer's protocol. Reverse transcription was performed with $1 \mu \mathrm{g}$ of RNA using a PrimeScript RT reagent kit and a gDNA Eraser kit (Takara, Dalian, China). We employed $\left(10 \mathrm{x}\left(\log _{2}{ }^{\Delta C T}\right)\right)^{-1}$ calculations to determine relative mRNA expression. Data were evaluated using the comparative count method and normalized to the corresponding $18 \mathrm{~S}$ ribosomal RNA value.

The primer sequences used were as follows: Wnt3a, forward, 5'-AATTTGGAGGAATGGTCTCTCGG-3' and reverse, 5'-CAGCAGGTCTTCACTTCACAG-3'; Wnt5a, forward, 5'-TCCGGACTACTGTGTGC-3' and reverse, 5'-AGC AGCACCAGTGAAAC-3'; Frizzled, forward, 5'-CGTACTGA GTGGAGTGTGTTTTG-3' and reverse, 5'-TGAGCTTTTCCA GTTTCTCTGTC-3'; c-Myc, forward, 5'-CGTCCTGGGAAG GGAGAT-3' and reverse, 5'-CGCTGCTATGGGCAAAGT-3'; MMP-7, forward, 5'-CACCACACTATTTTGAGGTCTTCC GCAG-3' and reverse, 5'-CATCCTAGGCTGAGGCTGGTAT GTTTTG-3'; 18S, forward, 5'-CGGCGACGACCCATTCG AAC-3' and reverse, 5'-GAATCGAACCCTGATTCCCC GTC-3'.

Western blot analysis. Protein samples were prepared from cancerous and adjacent tissues collected from eight patients 

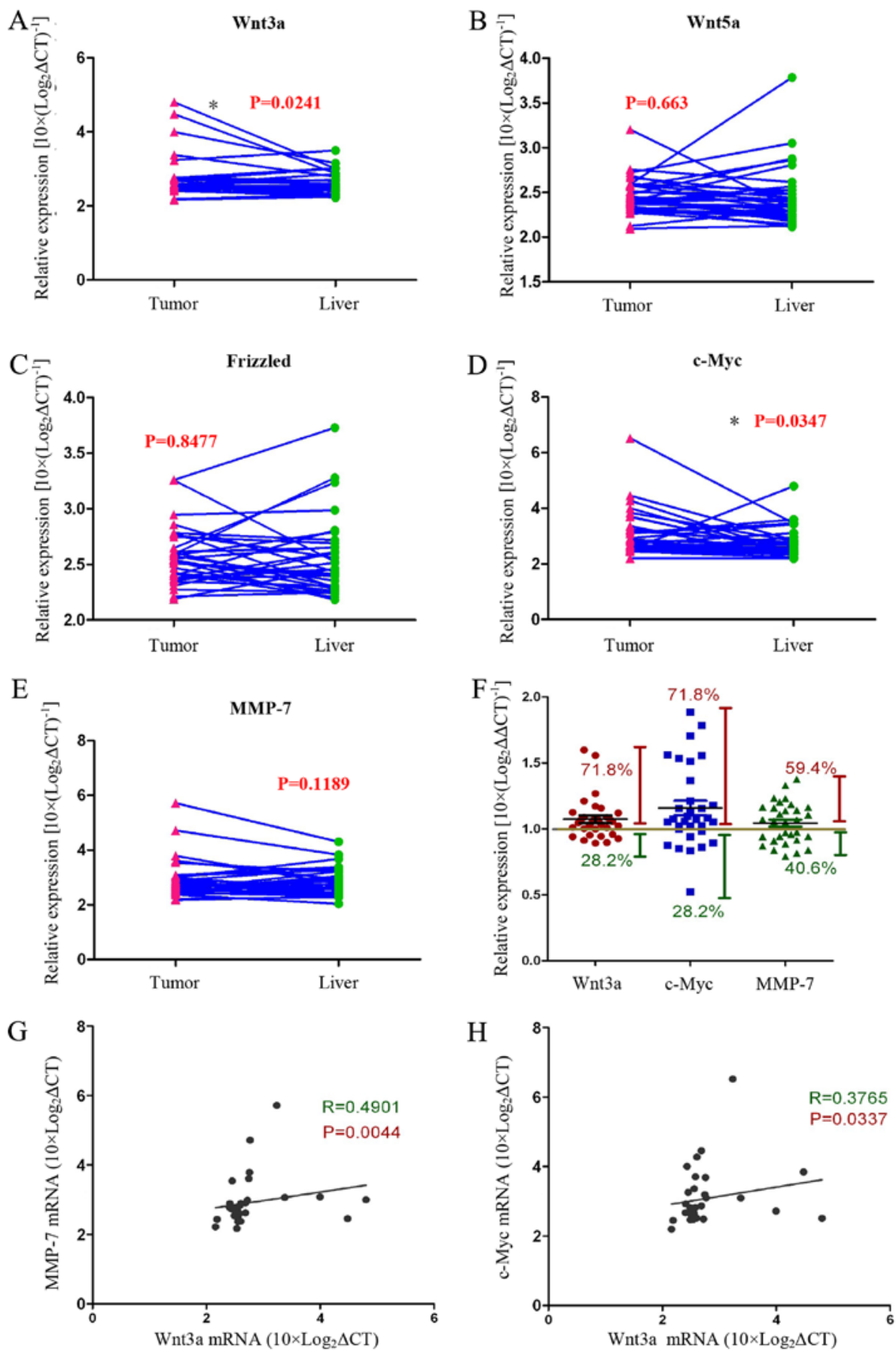

Figure 1. The Wnt3a pathway is activated in HCC tissues. Wnt3a pathway-related gene expression levels were measured with qRT-PCR (A-E). Wnt3a and c-Myc genes were expressed at higher levels in tumour tissues than in normal liver tissues [(A) $\mathrm{P}=0.0241$; (D) $\mathrm{P}=0.0347$; $\mathrm{n}=32$ )]. The Wnt5a, Frizzled and MMP-7 mRNA levels were not changed [(B, C and $\mathrm{E}) \mathrm{n}=32]$. In $71.8 \%$ of patients, Wnt3a and c-Myc expression was higher in tumour tissues than in normal liver tissues. However, no significant difference in MMP-7 expression between tumour tissues and normal liver tissues was observed (F). We analysed gene expression correlations using Pearson analysis and found that Wnt3a expression is positively correlated with MMP-7 and c-Myc expression [(G) R=0.4901, $\mathrm{P}=0.0044$; (H) $\mathrm{R}=0.3765, \mathrm{P}=0.0337]$, which suggests that the Wnt3a pathway is activated $\left({ }^{*} \mathrm{P}<0.05\right)$.

using Fastprep-24 Sample Preparation equipment (MP, USA), and the Wnt3a and Notch3 protein levels were detected. The cells were harvested and lysed in lysis buffer. Equal amounts of protein samples were loaded per well. Membranes containing protein blots were incubated in blocking buffer (5\% non-fat milk) for $1 \mathrm{~h}$ at room temperature, and then, the membranes were incubated with primary antibodies (Cell Signaling Technology) diluted 1:1,000 at $4^{\circ} \mathrm{C}$ overnight. Following a wash with TBS-T, the membranes were incubated with goat anti-rabbit secondary antibodies diluted 1:3,000 for $1 \mathrm{~h}$. Immunocomplexes were detected with enhanced chemiluminescence reagent.

Statistical analysis. The data were obtained from at least three independent experiments, and all analyses were performed with GraphPad version 5.0 software. The results were evaluated using Student's t-test, and Pearson correlation analyses were used to examine the correlation between two parameters. 

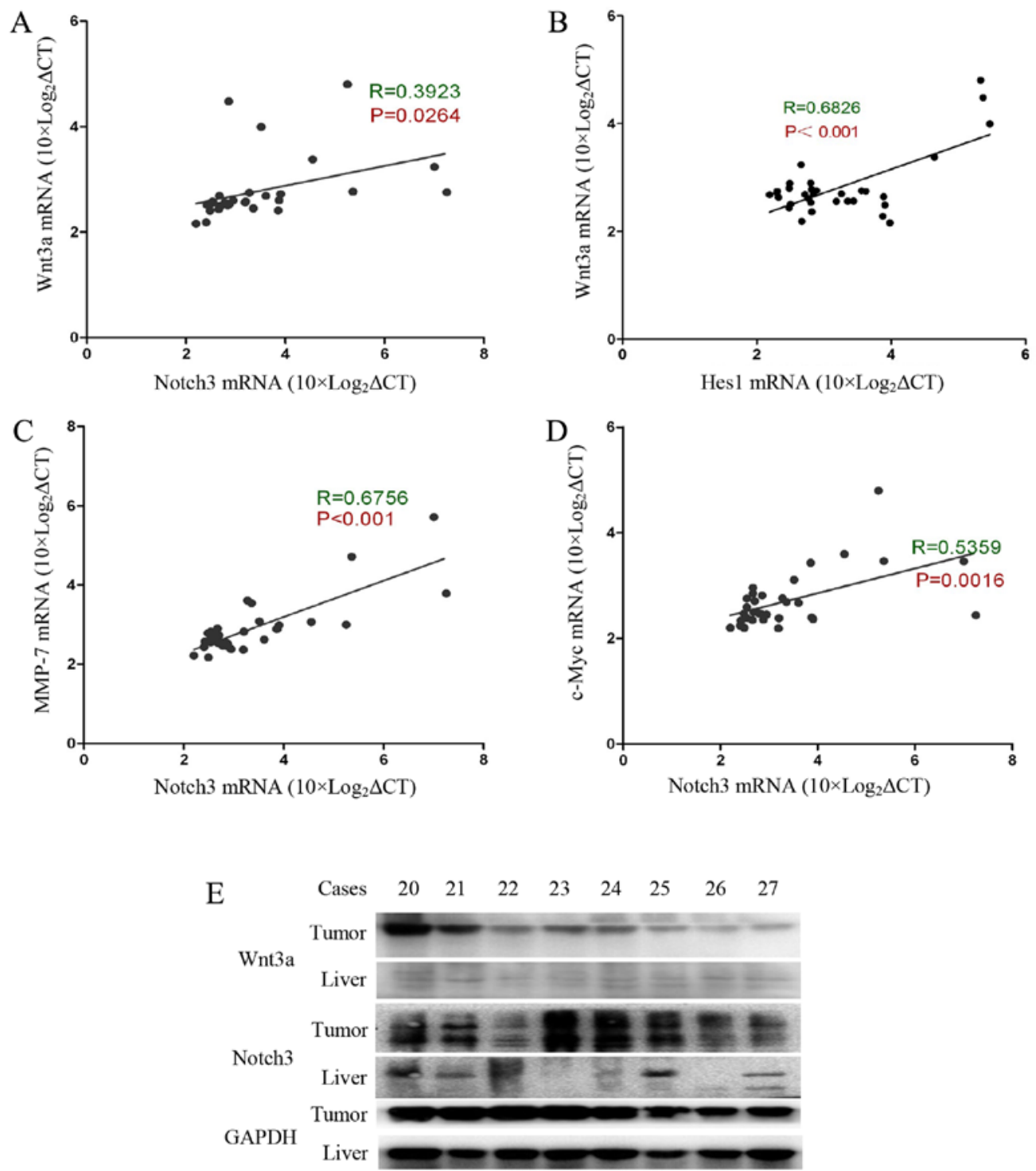

Figure 2. Wnt3a signalling activation is correlated with activation of the Notch3 signalling pathway in HCC. (A) Pearson correlation analysis revealed a correlation between Wnt3a and Notch3 and Hes1. Wnt3a is positively correlated with Notch3 and Hes1 [(A) $R=0.3923, P=0.0264 ;(B) R=0.6862, P<0.001]$. In addition, Notch3 expression is positively correlated with MMP-7 and c-Myc expression [(C) $R=0.6756, P<0.001$; (D) $R=0.5359, P=0.0016]$. We measured Wnt3a and Notch3 protein content with western blots. High Wnt3a expression was always accompanied by an increase in Notch3 expression (E).

The statistical significance levels were defined as $\mathrm{P}<0.05$, $\mathrm{P}<0.01$ and $\mathrm{P}<0.001$.

\section{Results}

The Wnt3a pathway is activated in HCC tissues. We assayed the expression of Wnt-related genes [Wnt3a, Wnt5a, Frizzled, c-Myc, and matrix metalloproteinase (MMP)-7] in HCC tissues and normal liver tissues with qRT-PCR. We found that the expression of Wnt3a and c-Myc genes was significantly increased in tumour tissues compared with normal liver tissues from the same patient (Fig. 1A-E). The data showed that 71.8\% of all cases $(n=32)$ expressed higher Wnt3a and Myc mRNA levels compared with normal liver tissue. In addition, MMP-7 gene expression was increased in tumour tissues compared with normal liver tissues in $>59.4 \%$ of the cases $(\mathrm{n}=32$ ) (Fig. 1F). We analysed the relationship between the Wnt3a and the Wnt pathway target genes c-Myc and MMP-7, and the data showed that they are positively correlated (Fig. 3G and H). These experimental data strongly support our view that the Wnt signalling pathway is activated in tumour tissues of HCC patients.

Wnt3a signalling activation is correlated with activation of the Notch3 signalling pathway in HCC. Our previous experimental results indicated that Notch3 and Hes1 gene expression levels are significantly increased in tumour tissue, and there is a positive correlation between Notch 3 and Hes1 gene expression levels (15). Interestingly, Wnt3a was found to be positively correlated with Notch $3(\mathrm{R}=0.39, \mathrm{P}=0.026)$ and Hes1 ( $\mathrm{R}=0.68, \mathrm{P}<0.001)$ (Fig. $2 \mathrm{~A}$ and $\mathrm{B})$. We also evaluated the correlation between Notch3 and the Wnt pathway target genes c-Myc and MMP-7. MMP-7 is involved in cancer cell invasion and metastasis processes $(16,17)$. c-Myc is a well-known gene that determines the cell capacity for self-renewal and proliferation (18). Our data show that Notch3 expression 

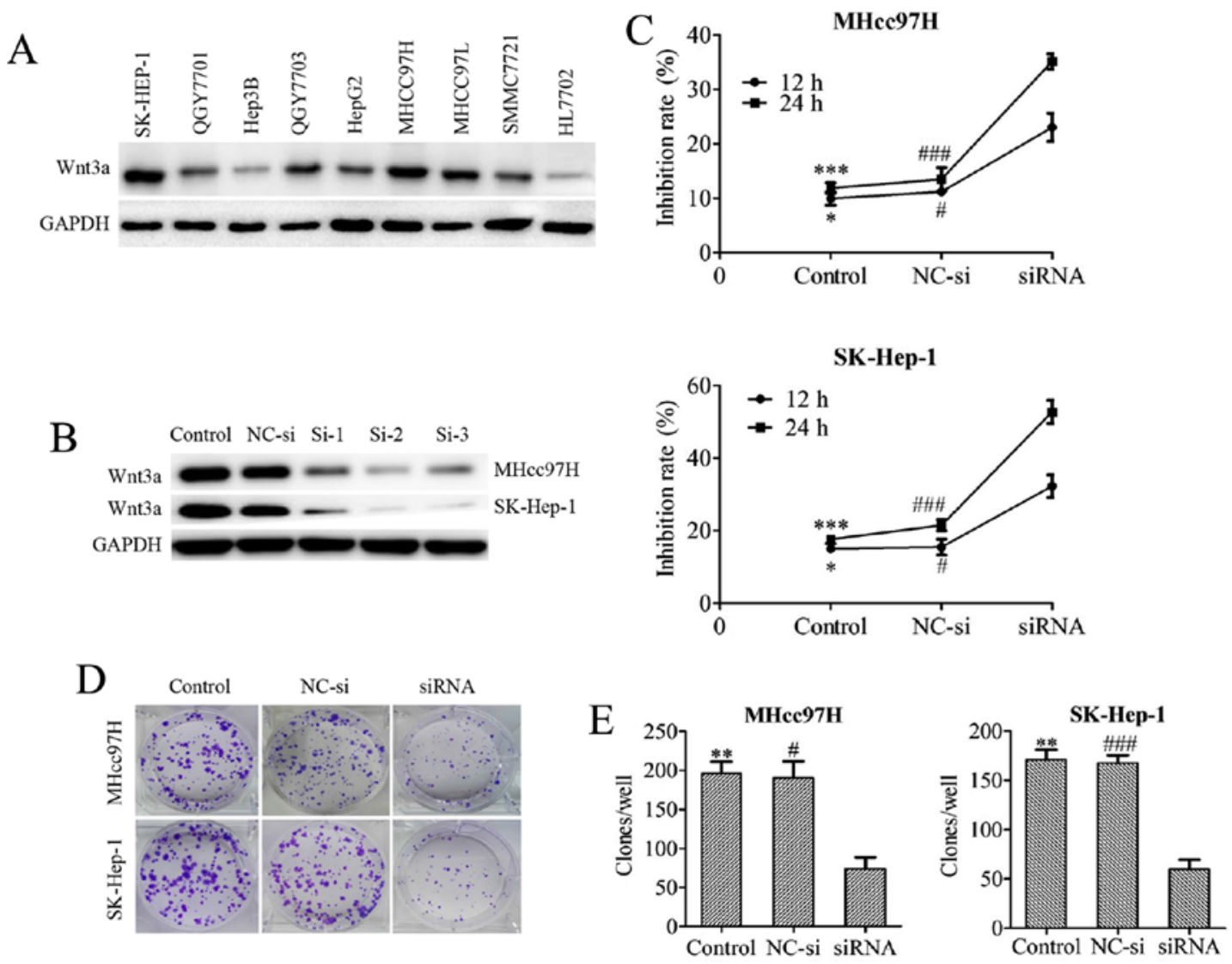

Figure 3. Downregulation of Wnt3a expression inhibits the viability of MHcc97H and SK-Hep-1 cells. The Wnt3a expression profiles of several HCC cell lines were screened by western blotting (A). The knockdown efficiency of 3 different Wnt3a siRNA sequences was tested in both MHcc97H and SK-Hep-1 cell lines by western blotting (B). The viability of cells treated with Wnt3a-siRNA for 12 and $24 \mathrm{~h}$ was determined using an MTT assay (C). The clonogenicity of cells after depletion of Wnt3a via siRNA interference was measured with a colony formation assay (D and E). The data are expressed as the means \pm SD of 3 independent experiments. ${ }^{*} \mathrm{P}<0.05,{ }^{* *} \mathrm{P}<0.01$ and ${ }^{* * *} \mathrm{P}<0.001$ between the indicated groups, control vs. siRNA; ${ }^{\# P}<0.05,{ }^{\# \#} \mathrm{P}<0.01$ and ${ }^{\# \# \# "} \mathrm{P}<0.001$ between the indicated groups, NC-si vs. siRNA; NC-si, negative control siRNA; si-1, 2, 3, Wnt3a-siRNA-1, 2, 3.

is significantly correlated with the expression of c-Myc and MMP-7 (Fig. 2C and D). In addition, we determined the Wnt $3 \mathrm{a}$ and Notch 3 protein contents in the tumour specimens and the accumulation of Wnt3a and Notch 3 in the tumour tissues (Fig. 2E). Based on these data, Wnt3a and Notch3 participate in the process of $\mathrm{HCC}$ carcinogenesis. Wnt3a may influence hepatocarcinoma cell invasion, metastasis and proliferation by targeting MMP-7 and c-Myc.

Downregulation of Wnt3a expression inhibits $\mathrm{MHcc} 97 \mathrm{H}$ and SK-Hep-1 cell viability. We first examined the baseline Wnt3a protein expression level in several hepatoma cell lines. Wnt3a exhibited higher expression levels in hepatoma cells compared with the normal liver cell line HL7702. Among the hepatoma cell lines, we found that $\mathrm{MHcc} 97 \mathrm{H}$ and SK-Hep-1 cells highly express Wnt3a (Fig. 3A). Therefore, we silenced Wnt3a mRNA expression via siRNA transfection in MHcc97H and SK-Hep-1 cells. The knockdown efficiency of 3 different Wnt3a siRNA sequences was tested in both MHcc97H and SK-Hep-1 cell lines, and the results were assessed with western blotting. Sequence 2 was chosen for use in subsequent experiments because of its excellent Wnt3a knockdown performance (Fig. 3B). The inhibitory effect of Wnt3a knockdown was measured with MTT assays after depletion of Wnt3a via siRNA interference in $\mathrm{MHcc} 97 \mathrm{H}$ and
SK-Hep-1 cells. Cell growth was potently inhibited in a timedependent manner (Fig. 3C). A colony formation assay was used to evaluate the ability of MHcc97H and SK-Hep-1 cells to form colonies after Wnt3a was downregulated by siRNA, and we found that Wnt3a inhibition reduced the colony-forming capability of the cells (Fig. 3D and E).

Silencing of Wnt3a expression induces cell cycle arrest by regulating cell cycle regulatory proteins. To investigate whether the anti-proliferative effect of decreased Wnt3a expression was triggered by cell cycle arrest, we examined cell cycle distribution using flow cytometry. We observed that after $24 \mathrm{~h}$ of siRNA interference, cell cycle progression was arrested in $\mathrm{MHcc} 97 \mathrm{H}$ cells at $\mathrm{G}_{0} / \mathrm{G}_{1}$ phase. Compared with the control and NC-si-transfected cells, the percentage of $\mathrm{G}_{0} / \mathrm{G}_{1}$ cells was increased significantly from 28.04 and 28.61 to $42.12 \%$. There was a similar effect on cell cycle distribution in SK-Hep-1 cells, and the percentage of $\mathrm{G}_{0} / \mathrm{G}_{1}$ cells increased significantly from 53.6 and 54.14 to $63.41 \%$. Analysis of this mechanism revealed that downregulation of Wnt3a was associated with downregulation of c-Myc and cyclin D1 expression and upregulation of p21 and p27 (Fig. 4).

Downregulation of Wnt3a expression suppresses $\mathrm{MHcc} 97 \mathrm{H}$ and SK-Hep-1 cell migration. To evaluate whether 
A
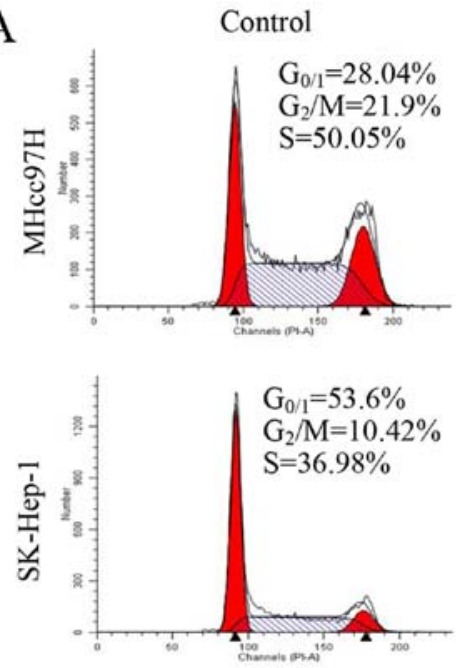
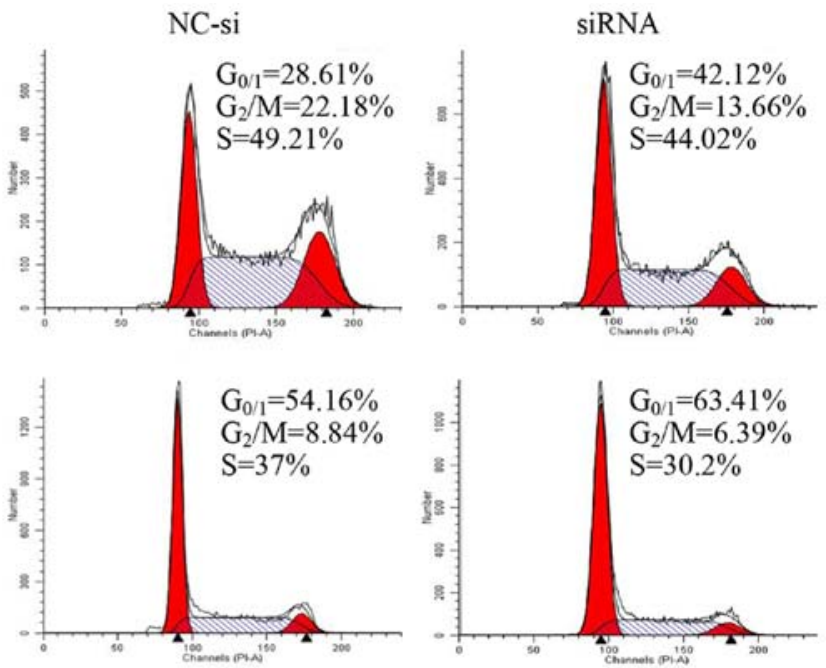

$\mathrm{B}$
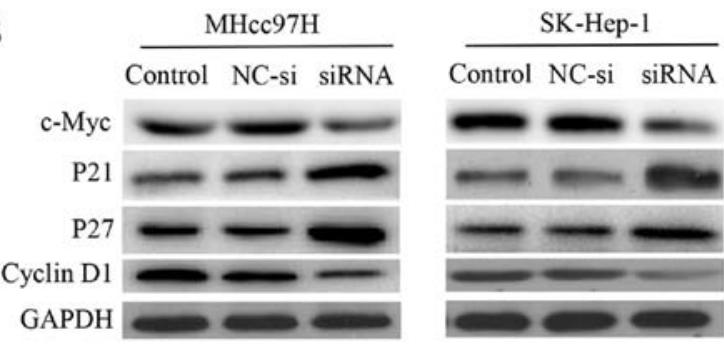

Figure 4. Silencing of Wnt3a expressions induces $\mathrm{G}_{0} / \mathrm{G}_{1}$ cell cycle arrest by regulating cell cycle regulatory proteins in $\mathrm{MHcc} 97 \mathrm{H}$ and $\mathrm{SK}-\mathrm{Hep}-1$ cells. Cell cycle profiles were measured with flow cytometry following Wnt3a-siRNA interference treatment for $24 \mathrm{~h}$ (A). Western blotting was performed to detect c-Myc, p21, p27, and cyclin D1 expression in MHcc97H and SK-Hep-1 cells; GAPDH was used as a loading control (B).

downregulation of $\mathrm{Wnt} 3 \mathrm{a}$ expression affects the metastatic ability of MHcc97H and SK-Hep-1 cells, we performed a wound healing assay. MHcc97H cell migration was significantly inhibited by Wnt3a knockdown. The size of the wound in the Wnt3a-siRNA group was $0.82 \pm 0.04 \mathrm{~mm}$, which was significantly larger than the wound size in either the control group $(0.32 \pm 0.04 \mathrm{~mm})$ or the NC-si-transfected group $(0.27 \pm 0.06 \mathrm{~mm})$ (Fig. $5 \mathrm{~A}$ and $\mathrm{B})$. Similar results were obtained for SK-Hep-1 cells; the size of the wound in the Wnt3a-siRNA group was $0.77 \pm 0.05 \mathrm{~mm}$, compared to $0.40 \pm 0.07 \mathrm{~mm}$ in the control group and $0.48 \pm 0.07 \mathrm{~mm}$ in the NC-si group (Fig. 5C and D). We used a Transwell Boyden chamber system with porous polycarbonate membranes to further quantify cell motility. The results were consistent with those of the wound healing assay. After MHcc97H cells were transfected with siRNA for $24 \mathrm{~h}$, the number of cells was reduced from $186.33 \pm 10.26$ and $180.67 \pm 10.07$ to $60.33 \pm 16.65$ compared with the control and NC-si groups. Similarly, the number of migrated SK-Hep-1 cells per field was reduced from $161.33 \pm 10.41$ and $157 \pm 11.14$ to $46.67 \pm 10.79$ (Fig. $5 \mathrm{E}$ and F). Taken together, our results support a role for Wnt3a in the migratory capability of MHCC97L and SK-Hep-l cells.

Downregulation of Wnt3a expression inhibits the invasion and adhesion of $\mathrm{MHcc} 97 \mathrm{H}$ and SK-Hep-1 cells. Transwell assay chambers with a Matrigel coating were used to evaluate tumour aggressiveness. The cells observed to degrade the Matrigel and pass through the membrane were counted, and the results revealed that the number of aggressive $\mathrm{MHcc} 97 \mathrm{H}$ cells was lower in the Wnt3a-siRNA group $(30 \pm 25.51)$ than in both the NC-si group $(113.33 \pm 8.14)$ and the control group (116 \pm 13.23$)$. Similar results were obtained for SK-Hep-1 cells (Wnt3a-siRNA, 38 \pm 13.45 ; NC-si, 149 \pm 14 ; control, 155.33 \pm 21.73 ) (Fig. 6A-C). Whether Wnt3a affects the adhesion ability of HCC cells was also investigated with an MTT assay. The data demonstrated that depletion of Wnt3a dramatically inhibited cell adhesion compared with the control group and NC-si group (Fig. 6D and E). Overall, these results support the proposal that downregulation of Wnt3a expression can significantly suppress the invasion and adhesion capability of MHCC97L and SK-Hep-l cells.

Wnt3a siRNA downregulates MMP-2, MMP-7 and MMP-9 protein expression in $M H c c 97 H$ and $S K-H E P-1$ cells. MMPs are known to play an important role in tumour metastasis $(16,19)$. A western blotting assay showed that the MMP-2/-9 protein levels in the Wnt3a-siRNA group were significantly lower than those in the other groups. Notably, the MMP-7 protein levels were also decreased after Wnt3a knockdown (Fig. 7). These results indicated that $\mathrm{Wnt} 3 \mathrm{a}$ promotes cell migration and invasion by regulating the expression of MMP-2/-7/-9.

Wnt3a-altered MMP expression is associated with the MAPK signalling pathway. We examined the expression of mitogen-activated protein kinase (MAPK) pathway-associated proteins, including the total protein levels of ERK1/2, p38, JNK and their phosphorylated forms (p-ERK1/2, p-p38, and $\mathrm{p}-\mathrm{JNK}$ ). The results demonstrated that downregulation of 
A

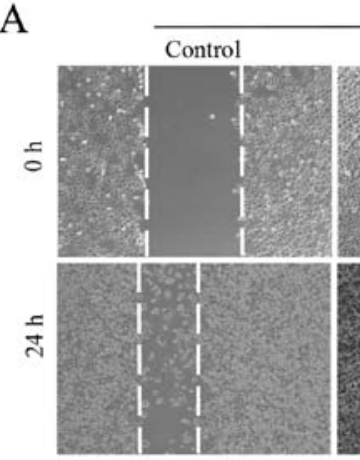

$\mathrm{C}$
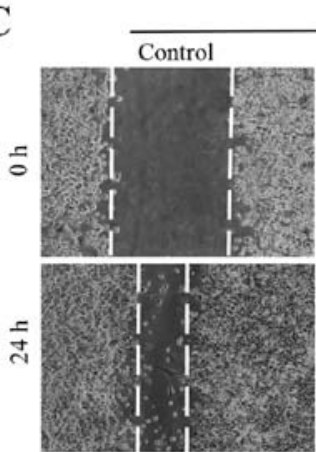

E

롱

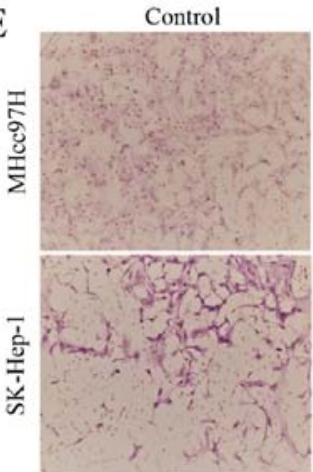

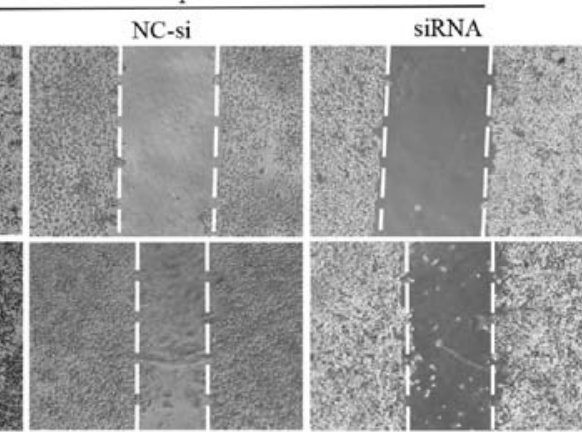

MHec $97 \mathrm{H}$

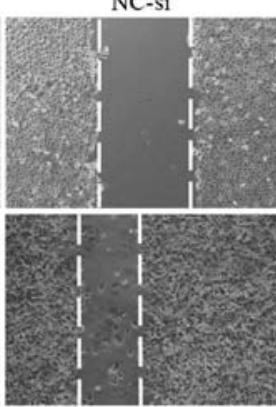

SK-Hep-1

NC-si

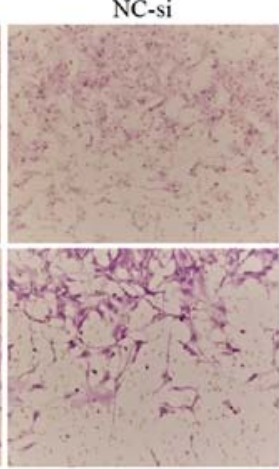

siRNA

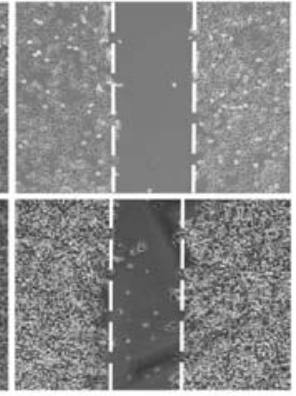

B

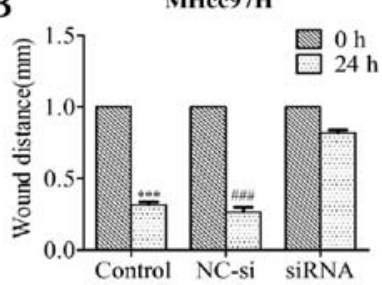

D
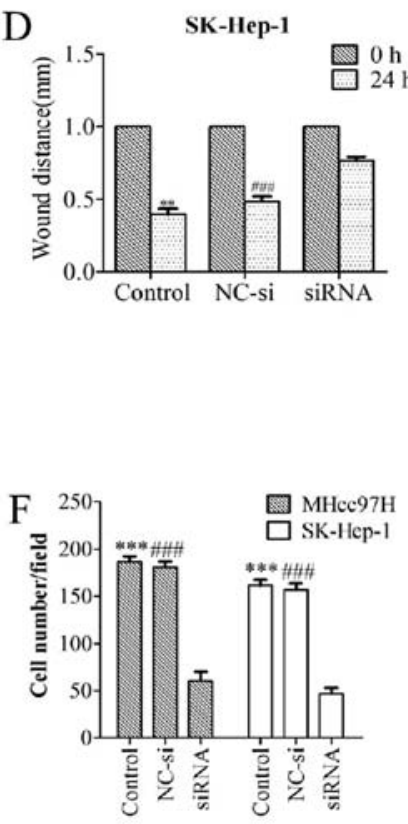

Figure 5. Downregulation of Wnt3a expression suppresses MHcc97H and SK-Hep-1 cell migration. Wound healing assays were performed in which the migration of cells into the wound was measured; images were captured at the indicated time-points. Downregulation of Wnt3a inhibited MHcc97H (A and B) and SK-Hep-1 (C and D) cell migration compared to the control and NC-si group. (A) Transwell Boyden chamber system was used to assay cell migration, and the representative images show that MHcc97H and SK-Hep-1 cell migration was decreased in the Wnt3a siRNA group compared to the control and NC-si groups ( $\mathrm{E}$ and $\mathrm{F})$.

Wnt3a had no effect on the protein levels of ERK1/2, p38 and JNK; however, the levels of the phosphorylated form were markedly decreased (Fig. 8).

\section{Discussion}

HCC patients have a higher mortality rate and a lower 5-year survival rate than patients with various other cancers $(20,21)$, mainly because of tumour cell invasion and metastasis. Thus, novel therapies that specifically inhibit these processes are critical. Some reports have suggested that the Wnt signalling pathway is activated and involved in tumour metastasis, but the detailed molecular mechanisms remain unclear. Our experiments attempted to explore the role of Wnt3a in pathogenesis and metastasis of HCC.

The expression of related genes in tumour specimens from HCC patients were evaluated with qRT-PCR. We found that expression of Wnt3a and the Wnt target gene c-Myc was upregulated. Wnt ligands can promote $\beta$-catenin nuclear translocation and enable $\beta$-catenin to interact with T-cell factor (TCF)/lymphoid enhancer factor (LEF) transcription factors to regulate gene expression, such as c-Myc, MMP-7 and cyclin D1 $(5,22,23)$. Wnt3a is a ligand in the Wnt signalling pathway. Driskell et al reported that Wnt3a knockout mice lacked LEF1 protein expression in submucosal gland (SMG) placodes, and confirmed that Wnt3a can directly transcriptionally regulate the LEF1 gene, which needs TCF4 to bind to the LEF1 promoter Wnt response region (24). Galceran et al showed that null mutations LEF1/TCF1 resulted in a defect in the formation of paraxial mesoderm, which was virtually identical to that seen in Wnt3a-deficient mice (25). Multiple studies reported that inhibition of Wnt3a expression reduced LEF/TCF activity and overexpression of wnt3a promoted LEF/TCF activity (26-28). In conclusion, Wnt3a can directly 

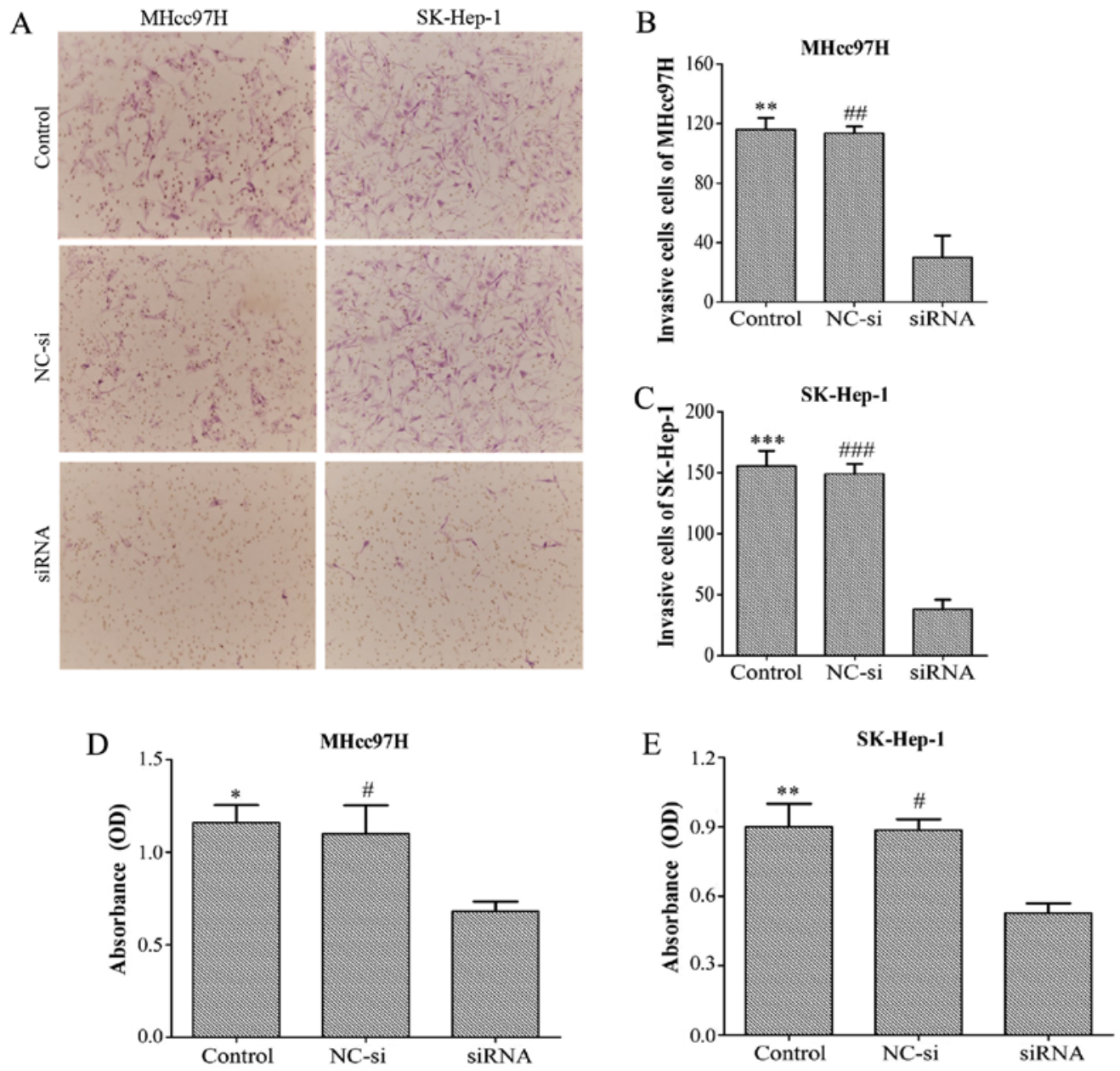

Figure 6. Downregulation of Wnt3a expression inhibits invasion and adhesion of MHcc97H and SK-Hep-1 cells. Cell invasion assays were performed using Transwell assays in which a Matrigel coating was added. Representative images showing that MHcc97H and SK-Hep-1 cell invasion was decreased in the Wnt3a siRNA group compared to the control and NC-si groups (A). Quantification of the number of invasive MHcc97H and SK-Hep-1 cells (B and C). Absorbance of the MHcc97H and SK-HEP-1 cells in an adhesion assay (D and E).

A

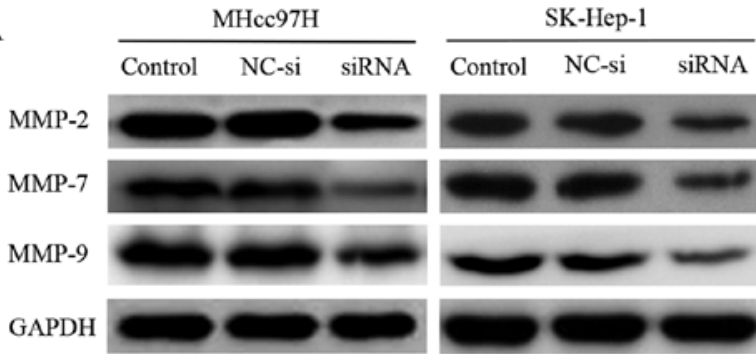

$\mathrm{C}$

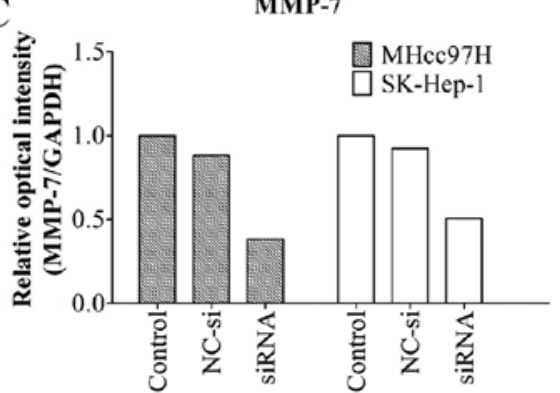

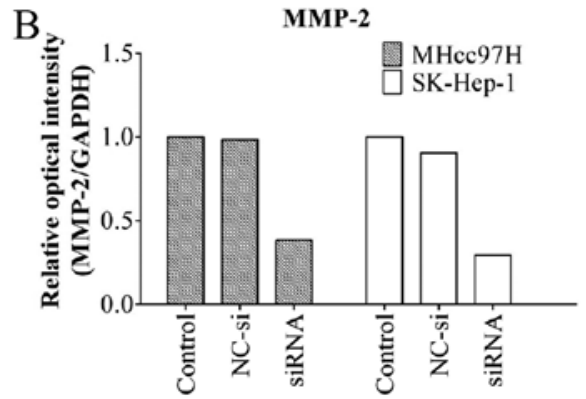

$\mathrm{D}$

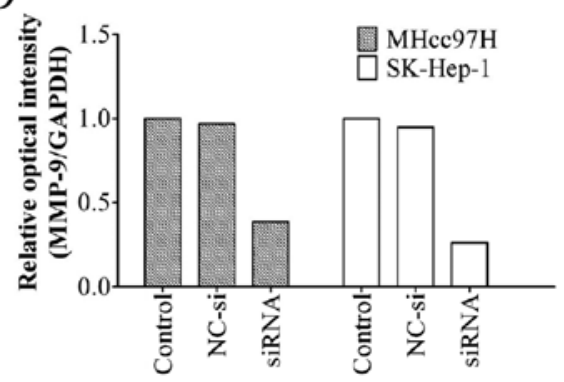

Figure 7. Downregulation of Wnt3a expression reduces the expression of MMP-2/-7/-9. The protein expression of MMPs (MMP-2/-7/-9) in MHcc97H and SK-Hep-1 cells after siRNA transfection for $24 \mathrm{~h}$ was examined by western blotting (A). Quantification of the expression levels of related proteins following transfection with siRNA (B-D). 
A

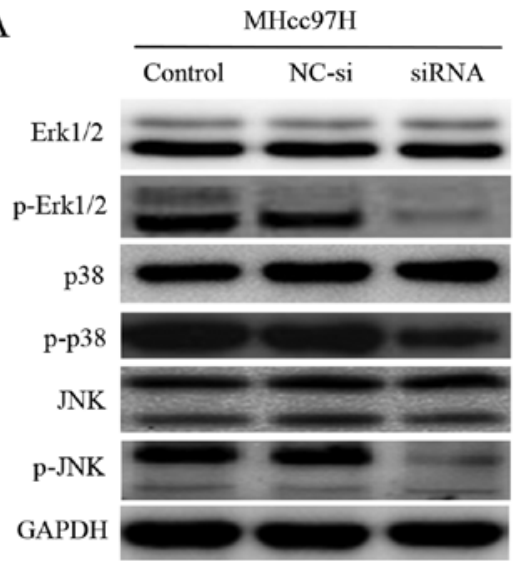

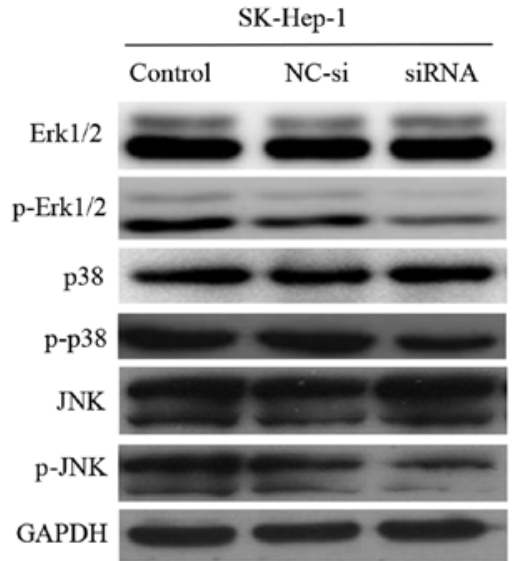
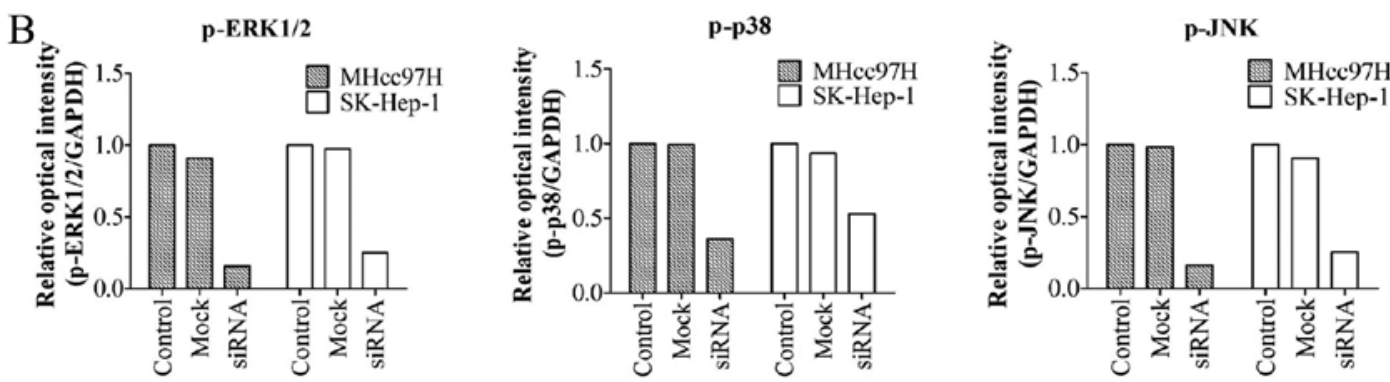

Figure 8. Wnt3a-altered MMP expression is associated with the MAPK signalling pathway. The expression of MAPK pathway proteins was examined by western blotting in MHcc97H and SK-Hep-1 cells after siRNA transfection for $24 \mathrm{~h}$ (A). Quantification of the expression levels of related proteins following transfection with siRNA (B).

transcriptionally regulate the LEF/TCF gene activity. Our data confirmed that Wnt3a plays an essential role in $\mathrm{HCC}$ progression. Activation of Wnt3a pathway is accompanied by higher expression of Notch3. Hyperactive Wnt3a could cause its target gene transcription activation. Stemness gene (c-Myc and MMP-7) expressed in HCC always suggest poor prognosis $(29,30)$. c-Myc is known as the stemness gene which determines the cell capacity of self-renewal and cell proliferation. MMPs are involved in the process of cancer cell invasion and metastasis $(16,17,30)$. The Wnt pathway is implicated in HCC pathogenesis through its interaction with many signalling pathways, including Hippo, AKT/PKB, ERK1/2, and NF- $\mathrm{B}$ signal transduction pathways (31-34). In addition, Sun et al (35) reported that Wnt and Notch1 pathways promote hepatitis B virus X protein-induced hepatocarcinogenesis. Our previous study found that Notch3 expression reflects differentiation properties and correlates with a poor prognosis in HCC and plays a role in modulating the stemness of HCC cells. Notch3 is a distinct biomarker of cancer stem cells in HCC progression. Therefore, HCC development may be associated with activation of multiple signalling pathways. Our data demonstrated that Wnt3a is positively correlated with Notch3 and Hes1 expression, and Notch3 is positively correlated with c-Myc and MMP-7 expression. Furthermore, we measured the protein levels of Wnt3a and Notch3 in the same specimen and confirmed that Wnt3a and Notch3 were highly expressed. Based on these data, we concluded that team work of Wnt pathway with Notch pathway contributed to regulate the maintenance of cellular stemness and recurrence as well as metastasis of HCC. However, their specific cooperation mechanism still needs to be clarified in the future.
Some studies have reported that the expression of $\mathrm{Wnt} 3 \mathrm{a}$ was inhibited by the inhibitors including AXIN2, DKK-1, NKD-1, $\beta$-TRCP and HS20 in liver cancers $(36,37)$, and these reports primarily elaborated the biological capability of these antagonists. However, the effect on Wnt pathway target gene and the capacity of proliferation and metastasis of the tumour cells were not further investigated after the reduction of Wnt3a. In our study, silencing of Wnt3a expression via siRNA transfection suppressed cell proliferation and induced $\mathrm{G}_{0} / \mathrm{G}_{1}$ cell cycle arrest in MHcc97H and SK-HEP-1 cells. Deregulation of cell cycle progression is a well-known and common feature of cancer (38). c-Myc is a key transcription factor involved in several cellular processes, including growth and proliferation in different cells $(39,40)$. The p21, p27 and Cyclin D1 proteins function as regulators of cell cycle progression through G1 and S phases (41-43). Thus, altered expression levels of these proteins indicate cell cycle arrest. Our data suggested that downregulating the expression of Wnt3a can reduce the expression of the target gene c-Myc. In addition, the protein levels of p21 and p27 were increased and cyclin D1 was decreased, indicating that Wnt3a siRNA treatment induce p21- and p27-dependent cell cycle arrest. In conclusion, these results clearly indicate that the Wnt3a pathway is involved in cell cycle progression in HCC cells.

Based on a functional analysis, we further examined the potential molecular mechanism of metastasis progression mediated by Wnt3a. MMPs, including MMP-2/-7/-9, play an important role in tumour metastasis $(9,17,19)$. Suppression of MMPs decreases cancer cell invasion and migration $(44,45)$. Accordingly, potential inhibitors of MMPs will undoubtedly be valuable in our attempts to treat HCC. Our studies 
demonstrated that inhibition of Wnt3a expression suppressed HCC cell invasion and metastasis by decreasing MMP-2/-7/-9 expression. The MAPK signalling pathway, including JNK, ERK $1 / 2$ and $\mathrm{p} 38$, has been reported to be the essential upstream signalling pathway that activates MMP expression and participates in regulation of cell migration and invasion in various cancers (46-48). To determine whether the downregulation of MMP expression is associated with MAPK signalling pathways, we assessed the effects of Wnt3a siRNA on JNK, ERK1/2, and p38 levels and those of their phosphorylated forms. The results demonstrated that cooperation of JNK, ERK1/2 and p38 plays a crucial role in Wnt3a-mediated cell migration and invasion in SK-Hep-1 and MHCC97L cells.

In conclusion, we confirmed that $\mathrm{Wnt} 3$ a plays an essential role in HCC progression. Activation of the Wnt3a pathway is accompanied by higher expression of Notch3. Furthermore, Wnt3a may be critical for HCC cell cycle and metastasis by regulating cell cycle regulatory proteins and the MAPK (p38, ERK1/2 and JNK) pathway. Wnt3a may represent a novel therapeutic target for treatment and inhibition of HCC metastasis.

\section{Acknowledgements}

This study was supported by the National Natural Science Funds 81041099 and Guangdong Province Natural Science Funds S2011010003750 (to M.L.). This study was also supported by the Institute of Hepatobiliary Surgery, Affiliated Hospital of Guangdong Medical University. We thank Professor Ningping Chen for advice and support.

\section{References}

1. Fitzmaurice C, Dicker D, Pain A, Hamavid H, Moradi-Lakeh M, MacIntyre MF, Allen C, Hansen G, Woodbrook R, Wolfe C, et al; Global Burden of Disease Cancer Collaboration: The global burden of cancer 2013. JAMA Oncol 1: 505-527, 2015.

2. Du ZG, Wei YG, Chen KF and Li B: Risk factors associated with early and late recurrence after curative resection of hepatocellular carcinoma: A single institution's experience with 398 consecutive patients. Hepatobiliary Pancreat Dis Int 13: 153-161, 2014.

3. Yang LY, Chang RM, Lau WY, Ou DP, Wu W and Zeng ZJ: Mesohepatectomy for centrally located large hepatocellular carcinoma: Indications, techniques, and outcomes. Surgery 156: 1177-1187, 2014.

4. Clevers $\mathrm{H}$ and Nusse R: Wnt/ $\beta$-catenin signaling and disease. Cell 149: 1192-1205, 2012.

5. Oishi N, Yamashita T and Kaneko S: Molecular biology of liver cancer stem cells. Liver Cancer 3: 71-84, 2014.

6. Cadigan KM and Nusse R: Wnt signaling: A common theme in animal development. Genes Dev 11: 3286-3305, 1997.

7. Pai SG, Carneiro BA, Mota JM, Costa R, Leite CA, Barroso-Sousa R, Kaplan JB, Chae YK and Giles FJ: Wnt/ beta-catenin pathway: Modulating anticancer immune response. J Hematol Oncol 10: 101, 2017.

8. Eterno V, Zambelli A, Villani L, Tuscano A, Manera S, Spitaleri A, Pavesi L and Amato A: AurkA controls self-renewal of breast cancer-initiating cells promoting wnt3a stabilization through suppression of miR-128. Sci Rep 6: 28436, 2016.

9. Lee MA, Park JH, Rhyu SY, Oh ST, Kang WK and Kim HN: Wnt3a expression is associated with MMP-9 expression in primary tumor and metastatic site in recurrent or stage IV colorectal cancer. BMC Cancer 14: 125, 2014.

10. Li C, Song G, Zhang S, Wang E and Cui Z: Wnt3a increases the metastatic potential of non-small cell lung cancer cells in vitro in part via its upregulation of Notch3. Oncol Rep 33: 1207-1214, 2015.

11. Tanaka M, Kuriyama S, Itoh G, Maeda D, Goto A, Tamiya Y, Yanagihara K, Yashiro M and Aiba N: Mesothelial cells create a novel tissue niche that facilitates gastric cancer invasion. Cancer Res 77: 684-695, 2017.
12. Leung WK, He M, Chan AW, Law PT and Wong N: Wnt/ $\beta$-catenin activates MiR-183/96/182 expression in hepatocellular carcinoma that promotes cell invasion. Cancer Lett 362: 97-105, 2015.

13. Peng YY, He YH, Chen C, Xu T, Li L, Ni MM, Meng XM, Huang $\mathrm{C}$ and Li J: NLRC5 regulates cell proliferation, migration and invasion in hepatocellular carcinoma by targeting the Wnt/ $\beta$-catenin signaling pathway. Cancer Lett 376: 10-21, 2016.

14. Liao CH, Yeh CT, Huang YH, Wu SM, Chi HC, Tsai MM, Tsai CY, Liao CJ, Tseng YH, Lin YH, et al: Dickkopf 4 positively regulated by the thyroid hormone receptor suppresses cell invasion in human hepatoma cells. Hepatology 55: 910-920, 2012.

15. Zhang Q, Lu C, Fang T, Wang Y, Hu W, Qiao J, Liu B, Liu J, Chen N, Li M, et al: Notch3 functions as a regulator of cell self-renewal by interacting with the $\beta$-catenin pathway in hepatocellular carcinoma. Oncotarget 6: 3669-3679, 2015.

16. Jia S, Lu J, Qu T, Feng Y, Wang X, Liu C and Ji J: MAGI1 inhibits migration and invasion via blocking MAPK/ERK signaling pathway in gastric cancer. Chin J Cancer Res 29: 25-35, 2017.

17. Shao Q, Luo X, Yang D, Wang C, Cheng Q, Xiang T and Ren G: Phospholipase C 11 suppresses cell migration and invasion of breast cancer cells by modulating KIF3A-mediated ERK1/2/ $\beta$-catenin/MMP7 signalling. Oncotarget 8: 29056-29066, 2017.

18. Cheng Q, Yuan F, Lu F, Zhang B, Chen T, Chen X, Cheng Y, Li N, Ma L and Tong T: CSIG promotes hepatocellular carcinoma proliferation by activating c-MYC expression. Oncotarget 6: 4733-4744, 2015 .

19. Okazaki I and Inagaki Y: Novel strategies for hepatocellular carcinoma based on MMPs science. Anticancer Agents Med Chem 12: 753-763, 2012.

20. DeSantis CE, Lin CC, Mariotto AB, Siegel RL, Stein KD, Kramer JL, Alteri R, Robbins AS and Jemal A: Cancer treatment and survivorship statistics, 2014. CA Cancer J Clin 64: 252-271, 2014.

21. Siegel R, Ma J, Zou Z and Jemal A: Cancer statistics, 2014. CA Cancer J Clin 64: 9-29, 2014.

22. Gai JQ, Sheng X, Qin JM, Sun K, Zhao W and Ni L: The effect and mechanism of bufalin on regulating hepatocellular carcinoma cell invasion and metastasis via $\mathrm{Wnt} / \beta$-catenin signaling pathway. Int J Oncol 48: 338-348, 2016.

23. Lian J, Tang J, Shi H, Li H, Zhen T, Xie W, Zhang F, Yang Y and Han A: Positive feedback loop of hepatoma-derived growth factor and $\beta$-catenin promotes carcinogenesis of colorectal cancer. Oncotarget 6: 29357-29374, 2015.

24. Driskell RR, Goodheart M, Neff T, Liu X, Luo M, Moothart C, Sigmund CD, Hosokawa R, Chai Y and Engelhardt JF: Wnt3a regulates Lef-1 expression during airway submucosal gland morphogenesis. Dev Biol 305: 90-102, 2007.

25. Galceran J, Fariñas I, Depew MJ, Clevers H and Grosschedl R:

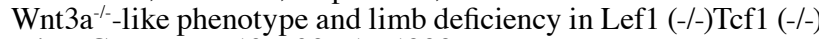
mice. Genes Dev 13: 709-717, 1999.

26. Qiang YW, Chen Y, Brown N, Hu B, Epstein J, Barlogie B and Shaughnessy JD Jr: Characterization of Wnt/beta-catenin signalling in osteoclasts in multiple myeloma. Br J Haematol 148: 726-738, 2010.

27. Wallace K, Marek CJ, Hoppler S and Wright MC: Glucocorticoiddependent transdifferentiation of pancreatic progenitor cells into hepatocytes is dependent on transient suppression of WNT signalling. J Cell Sci 123: 2103-2110, 2010.

28. Wang SH, Li N, Wei Y, Li QR and Yu ZP: $\beta$-catenin deacetylation is essential for WNT-induced proliferation of breast cancer cells. Mol Med Rep 9: 973-978, 2014.

29. Brabletz T, Jung A, Dag S, Hlubek F and Kirchner T: beta-catenin regulates the expression of the matrix metalloproteinase-7 in human colorectal cancer. Am J Pathol 155: 1033-1038, 1999.

30. Liu D, Nakano J, Ishikawa S, Yokomise H, Ueno M, Kadota K, Urushihara $\mathrm{M}$ and Huang CL: Overexpression of matrix metalloproteinase-7 (MMP-7) correlates with tumor proliferation, and a poor prognosis in non-small cell lung cancer. Lung Cancer 58: 384-391, 2007.

31. Erdal E, Ozturk N, Cagatay T, Eksioglu-Demiralp E and Ozturk M: Lithium-mediated downregulation of PKB/Akt and cyclin $\mathrm{E}$ with growth inhibition in hepatocellular carcinoma cells. Int J Cancer 115: 903-910, 2005.

32. Kim W, Khan SK and Yang Y: Interacting network of Hippo, Wnt $/ \beta$-catenin and Notch signaling represses liver tumor formation. BMB Rep 50: 1-2, 2017. 
33. Li J, Dai W, Xia Y, Chen K, Li S, Liu T, Zhang R, Wang J, Lu W, Zhou Y, et al: Astaxanthin inhibits proliferation and induces apoptosis of human hepatocellular carcinoma cells via Inhibition of NF- $\kappa$ B P65 and Wnt/ $\beta$-catenin in vitro. Mar Drugs 13: 6064-6081, 2015.

34. Wang R, Sun Q, Wang P, Liu M, Xiong S, Luo J, Huang H, Du Q, Geller DA and Cheng B: Notch and Wnt/ $\beta$-catenin signaling pathway play important roles in activating liver cancer stem cells. Oncotarget 7: 5754-5768, 2016.

35. Sun Q, Wang R, Luo J, Wang P, Xiong S, Liu M and Cheng B: Notch1 promotes hepatitis $\mathrm{B}$ virus $\mathrm{X}$ protein-induced hepatocarcinogenesis via Wnt/ $\beta$-catenin pathway. Int J Oncol 45: 1638-1648, 2014.

36. Gao W, Kim H, Feng M, Phung Y, Xavier CP, Rubin JS and Ho M: Inactivation of Wnt signaling by a human antibody that recognizes the heparan sulfate chains of glypican-3 for liver cancer therapy. Hepatology 60: 576-587, 2014.

37. Koch A, Waha A, Hartmann W, Hrychyk A, Schüller U, Waha A Wharton KA Jr, Fuchs SY, von Schweinitz D and Pietsch T: Elevated expression of Wnt antagonists is a common event in hepatoblastomas. Clin Cancer Res 11: 4295-4304, 2005.

38. Hanahan D and Weinberg RA: The hallmarks of cancer. Cell 100: $57-70,2000$

39. Akinyeke T, Matsumura S, Wang X, Wu Y, Schalfer ED, Saxena A, Yan W, Logan SK and Li X: Metformin targets c-MYC oncogene to prevent prostate cancer. Carcinogenesis 34: 2823-2832, 2013.

40. Li Y, Liu H,Lai C, Du X, Su Z and Gao S: The Lin28/let-7a/c-Myc pathway plays a role in non-muscle invasive bladder cancer. Cell Tissue Res 354: 533-541, 2013.
41. Bruyère $\mathrm{C}$ and Meijer L: Targeting cyclin-dependent kinases in anti-neoplastic therapy. Curr Opin Cell Biol 25: 772-779, 2013.

42. Wang ST, Ho HJ, Lin JT, Shieh JJ and Wu CY: Simvastatininduced cell cycle arrest through inhibition of STAT3/SKP2 axis and activation of AMPK to promote p27 and p21 accumulation in hepatocellular carcinoma cells. Cell Death Dis 8: e2626, 2017.

43. Yoon MK, Mitrea DM, Ou L and Kriwacki RW: Cell cycle regulation by the intrinsically disordered proteins $\mathrm{p} 21$ and $\mathrm{p} 27$. Biochem Soc Trans 40: 981-988, 2012.

44. Gao J, Ding F, Liu Q and Yao Y: Knockdown of MACC1 expression suppressed hepatocellular carcinoma cell migration and invasion and inhibited expression of MMP2 and MMP9. Mol Cell Biochem 376: 21-32, 2013.

45. Ha TY, Hwang S, Moon KM, Won YJ, Song GW, Kim N, Tak E, Ryoo BY and Hong HN: Sorafenib inhibits migration and invasion of hepatocellular carcinoma cells through suppression of matrix metalloproteinase expression. Anticancer Res 35: 1967-1976, 2015.

46. Guo JR, Li W, Wu Y, Wu LQ, Li X, Guo YF, Zheng XH, Lian XL, Huang HF and Chen YZ: Hepatocyte growth factor promotes proliferation, invasion, and metastasis of myeloid leukemia cells through PI3K-AKT and MAPK/ERK signaling pathway. Am J Transl Res 8: 3630-3644, 2016.

47. Luo Y, Wu JY, Lu M-H, Shi Z, Na N and Di J-M: Carvacrol alleviates prostate cancer cell proliferation, migration, and invasion through regulation of PI3K/Akt and MAPK signaling pathways. Oxid Med Cell Longev 2016: 1469693, 2016.

48. Yang Y, Ye Y, Qiu Q, Xiao Y, Huang M, Shi M, Liang L, Yang X and $\mathrm{Xu} \mathrm{H}$ : Triptolide inhibits the migration and invasion of rheumatoid fibroblast-like synoviocytes by blocking the activation of the JNK MAPK pathway. Int Immunopharmacol 41: 8-16, 2016. 